\title{
EFFECT OF SOIL-N APPLICATION AND FOLIAR NUTRITION WITH Zn AND Mn ON GROWTH, FLOWERING AND KEEPING QUALITY OF AMARYLLUS PLANTS (HIPPEASTRUM VITTATUM, HERB)
}

*Mostafa M. Rady, **Ebtsam M. Abdella and **Mahmoud A. Hassanain

* Botany Dept., Fac. Agric., Fayoum Branch, Cairo Univ., Egypt.

** Hort. Dept., Fac. Agric., Fayoum Branch, Cairo Univ., Egypt.

\section{ABSTRACT}

Apot experiment was conducted to study the effect of soil-N fertilization rates ( 2 and $4 \mathrm{~g}$ of ammonium nitrate/plant) and foliar spray with $\mathrm{Zn}$ and $\mathrm{Mn}$ at 2 concentrations (100 and $150 \mathrm{ppm}$ for each) on the vegetative growth, flowering and chemical composition of amaryllus (Hippeastrum vittatum, herb) plants. The obtained data showed that, fertilizing amaryllus plants with $4 \mathrm{~g}$ ammonium nitrate/plant significantly increased the vegetative growth characters i.e., plant height, number of leaves/plant, leaves area, fresh and dry weights of leaves, bulbs and the whole plant. Moreover, increased number of bulbs and bulblets/plant, bulb size and bulb dry weight. Flowering traits such as length of floral stalk, number of flowers on floral stalk, average dry weight of floral stalk and vase life of flowers were increased as well. The same rate $(4 \mathrm{~g})$ of $\mathrm{N}$-fertilization also led to the increase in the concentration of chlorophylls ( $a$ and b), carotenoids, total carbohydrates, anthocyanin in flowers at picking time and five days later, and leaves content of $\mathrm{N}$ and $\mathrm{P}$. The $2 \mathrm{~g}$ ammonium nitrate application/plant significantly increased both $\mathrm{K}$ and $\mathrm{Zn}$ contents in plant leaves. On the other side, leaf content of Mn and Fe was not affected with the 2 or $4 \mathrm{~g}$ ammonium nitrate rate. Foliar spray with the mixture of $\mathrm{Zn}$ and $\mathrm{Mn}$ at the rate $150 \mathrm{ppm}$ of each significantly increased all the above mentioned vegetative growth characters and flowering traits. The tested treatments also caused insignificant increase in the leaves content of chlorophylls ( $a$ and b), carotenoids, total carbohydrates, flowers content of anthocyanin at picking time and 5 days later, and accumulation of $\mathrm{N}$ and $\mathrm{K}$. The greatest accumulation of $\mathrm{P}$ occured when plants were sprayed with $\mathrm{Mn}$ at $150 \mathrm{ppm}$. On the contrary, the highest value of Fe was observed in plants sprayed with $\mathrm{Zn}$ and $\mathrm{Mn}$ at the concentrations of 150 and 100 ppm, respectively. Regarding the combined effect of soil $\mathrm{N}$ application and foliar spray with $(\mathrm{Zn}+\mathrm{Mn})$, the results revealed that, fertilizing the amaryllus plants with $4 \mathrm{~g}$ ammonium nitrate and foliar application with mixture of $\mathrm{Zn}$ and $\mathrm{Mn}$ at $150 \mathrm{ppm}$ concentration for each significantly increased all vegetative growth characters, floral parameters, leaves content of chlorophylls ( $\mathrm{a}$ and $\mathrm{b}$ ), carotenoids, total carbohydrates, N, P and flowers content of anthocyanin at the time of picking or five days later. The greatest plant content of $\mathrm{K}$ was found when a plant recivied $2 \mathrm{~g}$ of $\mathrm{N}$ and sprayed with $\mathrm{Zn}$ and $\mathrm{Mn}$ at $150 \mathrm{ppm}$ for each. Providing amaryllus plants with $2 \mathrm{~g}$ of ammonium nitrate and foliar spraying with only $\mathrm{Zn}$ at $150 \mathrm{ppm}$ resulted in the greatest leaf content of $\mathrm{Zn}$ and Fe.

Fayoum J. Agric. Res. \& Dev., Vol.19, No.2, July, 2005 
Mostafa M. Rady, et al.

Key words: Amaryllus, Hippeastrum vittatum, Soil-N Application, Foliar Znand Mn-Nutrition, Vegetative growth, flowering, Chemical composition.

\section{INTRODUCTION}

Amaryllus (Hippeastrum vittatum, Herb), Family amaryllidaceae is an attractive flowering ornamental bulb, native to South America. It is grown in Egypt both outdoors, as flower bed impact and inborders, as well as, for cut flowers and indoors, as a foliage and flowering pot plant in a time where there is nearly a lack of other flowers. The inflorescences emerges under Egypt temperature in mid-April and lasts for a relatively short period (2-4 weeks). It consists of four large and colorific trumpet-like flowers, that last about two weeks with only one or two inflorescences for a vase. Fertilizing in general and with nitrogen in particular has been known as a vital step in steping up the growth and flowering of many ornamental plants. Dahya et al. (1999), Chadha et al. (1999), Hameed and Sekar (1999), Ram et al. (1999) and Broschat and Moore (2001) working on marigold plants and El-Gendy et al. (2001) working on Ocimum bacilicum declared that, increasing $\mathrm{N}$-application up to a particular level, consistently increase vegetative growth parameters and flowering traits as well as the total carbohydrates and nitrogen content of plant.

Although micronutrients are needed in relatively very small quantities for good plant growth, their deficiencies cause great disorders in the physiological and metabolic processes of plant (Kanwer and Dhingra, 1962). Zinc and manganese were reported to stimulate the growth of various plants due to their enhancing effect on most metabolic processes such as carbohydrates, protein, phosphate RNA and ribosome formation (Price $\boldsymbol{e} \boldsymbol{t}$ al., 1972 and Bidwell, 1980), in chlorophyll formation and nucleic acid metabolism (Mohr and Schopfer, 1995) and in oxidation-reduction processes (Mengel and Kirkby, 1982). They announced also that, high $\mathrm{pH}$ and low organic matter in the soil considerably reduce the availability of most microelements. The intensive cropping without adequate conservation of soil fertility through balanced fertilization may be responsible for deficiencies of most nutrients, especially microelements in plants grown in Egypt (Sillanpaa, 1982). It was found by several investigators that, micronutrients play an important role in improving the vegetative growth and flowering of several ornamental plants (Selim and El-Tantawy, 1993) on gerbera, (El-Deeb, 1999) on Philodendron scandatum, (Refaat and Balbaa, 2001) on lemongrass plants and (Selim et al., 2001) on Calendula officinalis. They concluded that, spraying plants with $\mathrm{Zn}$ and $\mathrm{Mn}$ increase all vegetative growth characters and flowering. The effect of $\mathrm{Zn}$ or $\mathrm{Mn}$ on the chemical composition of plants was reported by many investigators (El-Deeb, 1999) on Philodendron scandatum and (Selim et al., 2001) on Calendula officinalis. They found that, $\mathrm{Zn}$ and $\mathrm{Mn}$ foliar sprays increase the plant content of chlorophyll $a$ or $b$ and total carotenoids. Refaat and Balbaa (2001) on lemongrass plants indicated that $\mathrm{Zn}$ and $\mathrm{Mn}$ increase chlorophylls ( $\mathrm{a}$ and $\mathrm{b}$ ), carotenoids, macronutrients (N, P and $\mathrm{K}$ ) and the micronutrient cations (Fe, $\mathrm{Mn}$ and $\mathrm{Zn}$ ) while decrease total carbohydrates.

Fayoum J. Agric. Res. \& Dev., Vol.19, No.2, July, 2005 
EFFECT OF SOIL-N APPLICATION AND FOLIAR NUTRITION......81

Objective of the present work is to investigate the effect of soil-N application and foliar spray with $\mathrm{Zn}$ and $\mathrm{Mn}$ and their interaction on the vegetative growth, flowering and chemical composition of amaryllus (Hippeastrum vittatum, Herb) plant.

\section{MATERIALS AND METHODS}

A pot experiment was conducted during the two successive seasons of 2003/2004 and 2004/2005 at the Experimental Farm of the Faculty of Agriculture, Fayoum, Cairo University, in order to study the effect of soil-N application and foliar spray with $\mathrm{Zn}$ and $\mathrm{Mn}$ and their interaction on the vegetative growth, flowering traits and bulbs yield of amaryllus (Hippeastrum vittatum, Herb) plants. Clay pots, $30 \mathrm{~cm}$ diameter, were filled with equal amounts of air dried clay soil mixed with sand at a ratio 1:1 by weight. Uniform sizes of amaryllus bulbs were utilized. One bulb was sown in each pot on August 25, 2003 and 2004. Samples of the used soil were analyzed according to Black (1965). Results of analysis are given in Table (1).

Table 1. Some physical and chemical properties of the experimental soil.

\begin{tabular}{|c|c|c|}
\hline Properties & $2003 / 2004$ & $2004 / 2005$ \\
\hline \multicolumn{3}{|l|}{ Particle size distribution: } \\
\hline Sand \% & 32.100 & 32.270 \\
\hline Silt\% & 32.070 & 31.410 \\
\hline Clay\% & 35.830 & 36.320 \\
\hline Texture grade & Sandy clay & Sandy clay \\
\hline $\begin{array}{l}\text { Hydraulic conductivity } \\
\left(\mathrm{cm}^{3} / \mathrm{hr}\right)\end{array}$ & 00.027 & 00.029 \\
\hline \multicolumn{3}{|l|}{ Chemical properties: } \\
\hline Calcium carbonate \% & 4.800 & 4.600 \\
\hline Organic matter\% & 1.250 & 1.280 \\
\hline Total nitrogen \% & 0.062 & 0.066 \\
\hline $\mathrm{EC}_{\mathrm{e}}(\mathrm{dS} / \mathbf{m})$ & 2.900 & 2.600 \\
\hline PH of paste extract & 7.500 & 7.300 \\
\hline \multicolumn{3}{|l|}{$\begin{array}{l}\text { Available nutrients } \quad(\mathrm{mg} / \mathrm{kg} \\
\text { soil): }\end{array}$} \\
\hline $\mathbf{P}$ & 23.00 & 22.00 \\
\hline $\mathbf{K}$ & 96.80 & 99.26 \\
\hline $\mathbf{Z n}$ & 00.81 & 00.80 \\
\hline $\mathbf{F e}$ & 03.64 & 03.71 \\
\hline Mn & 08.03 & 07.89 \\
\hline
\end{tabular}

Fertilization treatments included the combinations of two $\mathrm{N}$ rates: 2 and $4 \mathrm{~g}$ ammonium nitrate $(33.5 \% \mathrm{~N})$ /plant, two concentrations of $\mathrm{Zn}$ (100 and $150 \mathrm{ppm})$ and two of Mn (100 and $150 \mathrm{ppm})$ applied as elemental zinc (65\% $\mathrm{Zn})$ and elemental manganese $(65 \% \mathrm{Mn})$, respectively. Nitrogen fertilizer was added as a soil application in two equal portions; at November, $15^{\text {th }}$ and 15 days later. Zinc and manganese were applied as 3 foliar sprays at 2 weeks intervals starting from November 8 , in both seasons, respectively. The experimental layout was a factorial experiment in a randomized complete blocks design with four replications for each experimental unit. Equal doses of potassium sulphate and calcium superphosphate; were added to each pot at the

Fayoum J. Agric. Res. \& Dev., Vol.19, No.2, July, 2005 
rate $3 \mathrm{~g} / \mathrm{pot}$. All other agromanagements for the production of amaryllus flowers were achieved whenever it was necessary. When the $1^{\text {st }}$ flower of floal stalk, in each pot, seemed to be open, the following measurements were performed:

1. Vegetative growth characters in terms of plant height, number of leaves, leaves area/plant, dry weight of plant tops and whole plant (plant tops+bulbs), number of bulbs and bulblets, bulb fresh weight and bulb size.

2. Flowering traits expressed as length, number of flowers and fresh weight /floal stalk. Vase life of flowers as number of days starting from opening of the $1^{\text {st }}$ flower till wilting the last one was considered.

3. Chemical composition of plant leaves: concentrations of chlorophylls (a and $b$ ) and carotenoids in fresh leaves at the beginning of flowering were determined according to the methods described by Welburn and Lichtenthaler (1984). Total carbohydrates in leaves was colorimetrically estimated as outlined by Herbert $\boldsymbol{e t}$ al. (1971). Leaf $\mathrm{N}$ was determined using Orange $G$ dye according to Hafez and Mikkelsen (1981). For P,K, Mn, Zn and Fe determinaton, the wet digestion of $0.1 \mathrm{~g}$ of fine dry material of leaves of each treatment was done with sulphuric and perchloric acids as described by Piper (1947). Phosphorus was estimated colorimetrically by the method as outlined by King (1951) after extraction according to Olsen and Sommors (1982). Potassium was determined by Flame Photometer (Gallenkamp Co., England) as described by Brown and Lilliand (1966). Leaf $\mathrm{Mn}, \mathrm{Zn}$ and $\mathrm{Fe}$ were determined using atomic absorption spectrophotometer as outlined by Parkinson and Allen (1975). All the obtained results were statistically analyzed and comparisons among means of the different treatments were achieved using the least significant differences (LSD) at $p=0.05$ as illustrated by Snedecor and Cochran (1980).

\section{RESULTS AND DISCUSSION}

\section{Vegetative Growth Characters:}

Data illustrated in Tables (2a, $2 \mathrm{~b}$ and $2 \mathrm{c}$ ) exhibit that, $\mathrm{N}$-application to the growing Hippeastrum vittatum plants at the rate $4 \mathrm{~g} \mathrm{NH}_{4} \mathrm{NO}_{3} /$ pot was superior and associated significantly with higher mean magnitudes for plant height, number of leaves/ plant, leaves area/plant, dry weight for each of the plant tops, bulbs and whole plant (plant tops+bulbs), average number of bulbs and bulblets/pot, bulb size and bulb fresh weight than those grown with the lower level of $\mathrm{N}$ ( $2 \mathrm{~g}$ ammonium nitrate/plant). The obtained data matched well with the findings of Dahya et al. (1999), Chadha et al. (1999), Hameed and Sakar (1999), Ram et al. (1999) and Broschat and Moore (2001) on marigold plants and El-Gendy et al. (2001) on Ocimum basilicum. They reported that increased $\mathrm{N}$-application up to a particular level, consistency increased vegetative growth parameters.

Foliar spray of amaryllus plants with the mixture of $\mathrm{Zn}$ and $\mathrm{Mn}$ at 150 ppm concentration, for each, significantly or unsignificantly increased all the studied vegetative growth parameters followed by the mixture of $150 \mathrm{ppm} \mathrm{Zn}$ and $100 \mathrm{ppm} \mathrm{Mn}$ (tables 2a, 2b and 2c). The favourable effect of $\mathrm{Zn}$ and $\mathrm{Mn}$ may be due to the role of $\mathrm{Zn}$ in promotion of enzymes activity and the internal growth regulators which may be associated with plant growth. The obtained results are in harmony with the findings of Abed et al. (1987) on Pisum

Fayoum J. Agric. Res. \& Dev., Vol.19, No.2, July, 2005 
EFFECT OF SOIL-N APPLICATION AND FOLIAR NUTRITION.....8 83 sativum, Selim and El-Tantawy (1993) on gerbera, El-Deeb (1999) on Philodendron scandens, Refaat and Balbaa (2001) on lemongrass plants and Selim et al. (2001) on Calendula officinalis, who retrieved that spraying the plants with $\mathrm{Zn}$ or Mn increased all vegetative characters.

Concerning the interaction between the different levels of ammonium nitrate fertilizer and foliar sprays of $\mathrm{Zn}$ or $\mathrm{Mn}$ and their mixture on vegetative growth varied between the two seasons. The significant response was obvious in one out of the two experimental seasons (tables $2 \mathrm{a}, 2 \mathrm{~b}$ and $2 \mathrm{c}$ ). Comparison among all treatments indicated that the combination of $4 \mathrm{~g}$ ammonium nitrate/plant with the mixture of $\mathrm{Zn}$ and $\mathrm{Mn}$ at the concentration of $150 \mathrm{ppm}$ for each proved to be the best for all vegetative growth parameters.

Table 2a. Effect of soil $\mathrm{N}$-application and foliar nutrition with $\mathrm{Zn}$ and $\mathrm{Mn}$ on vegetative growth characters of amaryllus plants during the two successive seasons 2003/2004 and 2004/2005.

\begin{tabular}{|c|c|c|c|c|c|c|c|c|c|}
\hline \multirow{2}{*}{$\begin{array}{l}\text { Character } \\
\text { Treatment }\end{array}$} & \multicolumn{3}{|c|}{$\begin{array}{l}\text { Plant height } \\
(\mathbf{c m})\end{array}$} & \multicolumn{3}{|c|}{ No. of leaves/plant } & \multicolumn{3}{|c|}{$\begin{array}{c}\text { Leaves area/plant } \\
\left(\mathrm{dm}^{2}\right)\end{array}$} \\
\hline & $\mathbf{N}_{1}$ & $\mathbf{N}_{2}$ & $\underset{\text { (B) }}{\text { Mean }}$ & $\mathbf{N}_{1}$ & $\mathbf{N}_{2}$ & $\underset{(\mathbf{B})}{\operatorname{Mean}}$ & $\mathbf{N}_{1}$ & $\mathbf{N}_{2}$ & $\begin{array}{c}\text { Mean } \\
(\mathbf{B})\end{array}$ \\
\hline \multicolumn{10}{|c|}{$2003 / 2004$} \\
\hline $\mathbf{Z n}_{0}+\mathbf{M n}_{0}$ & 42.67 & 43.67 & 43.17 & 6.33 & 6.67 & 6.50 & 6.86 & 7.36 & 7.11 \\
\hline $\mathrm{Zn}_{0}+\mathbf{M n}_{1}$ & 44.67 & 46.00 & 45.34 & 6.67 & 7.33 & 7.00 & 7.30 & 8.00 & 7.65 \\
\hline $\mathbf{Z n}_{0}+\mathbf{M n}_{2}$ & 47.33 & 48.33 & 47.83 & 7.00 & 7.67 & 7.34 & 7.70 & 8.24 & 7.97 \\
\hline $\mathrm{Zn}_{1}+\mathrm{Mn}_{0}$ & 45.67 & 47.33 & 46.50 & 7.00 & 7.33 & 7.17 & 7.55 & 8.10 & 7.83 \\
\hline $\mathbf{Z n}_{1}+\mathbf{M n}_{1}$ & 49.33 & 50.67 & 50.00 & 7.33 & 7.67 & 7.50 & 8.10 & 8.24 & 8.17 \\
\hline $\mathbf{Z n}_{1}+\mathbf{M n}_{2}$ & 52.33 & 53.33 & 52.83 & 7.67 & 7.67 & 7.67 & 8.40 & 8.44 & 8.42 \\
\hline $\mathrm{Zn}_{2}+\mathrm{Mn}_{0}$ & 50.67 & 52.00 & 51.34 & 7.67 & 7.67 & 7.67 & 8.24 & 8.30 & 8.27 \\
\hline $\mathrm{Zn}_{2}+\mathrm{Mn}_{1}$ & 53.67 & 54.33 & $\mathbf{5 4 . 0 0}$ & 8.00 & 8.00 & 8.00 & 8.66 & 8.85 & 8.76 \\
\hline $\mathrm{Zn}_{2}+\mathrm{Mn}_{2}$ & 55.67 & 56.67 & 56.17 & 8.00 & 8.33 & 8.17 & 8.78 & 9.26 & 9.02 \\
\hline Mean (A) & 49.11 & 50.26 & & 7.30 & 7.59 & & $\mathbf{7 . 9 5}$ & 8.31 & \\
\hline \multicolumn{10}{|l|}{$\operatorname{LSD}_{(0.05)}:$} \\
\hline (A) & & 1.06 & & & 0.21 & & & 0.33 & \\
\hline (B) & & 1.59 & & & 0.32 & & & 0.42 & \\
\hline$(\mathbf{A}) \times(\mathbf{B})$ & & 2.11 & & & 0.40 & & & 0.60 & \\
\hline \multicolumn{10}{|c|}{$2004 / 2005$} \\
\hline $\mathbf{Z n}_{0}+\mathbf{M n}_{0}$ & 43.33 & 44.00 & 43.67 & 6.00 & 6.33 & 6.17 & 6.60 & 6.96 & 6.78 \\
\hline $\mathbf{Z n}_{0}+\mathbf{M n}_{1}$ & 46.00 & 46.33 & 46.17 & 6.33 & 6.67 & 6.50 & 6.96 & 7.34 & 7.15 \\
\hline $\mathrm{Zn}_{0}+\mathrm{Mn}_{2}$ & 48.33 & 49.00 & 48.67 & 7.00 & 7.33 & 7.17 & 7.70 & 8.06 & 7.88 \\
\hline $\mathrm{Zn}_{1}+\mathrm{Mn}_{0}$ & 47.33 & 47.67 & 47.50 & 6.67 & 7.00 & 6.84 & 7.34 & 7.70 & 7.52 \\
\hline $\mathbf{Z n}_{1}+\mathbf{M n}_{1}$ & 48.67 & 51.00 & 49.84 & 7.00 & 7.33 & 7.17 & 7.70 & 8.06 & 7.88 \\
\hline $\mathrm{Zn}_{1}+\mathrm{Mn}_{2}$ & 52.33 & 53.67 & $\mathbf{5 3 . 0 0}$ & 7.33 & 7.67 & 7.50 & 8.06 & 8.44 & 8.25 \\
\hline $\mathrm{Zn}_{2}+\mathrm{Mn}_{0}$ & 50.00 & 52.00 & 51.00 & 7.00 & 7.67 & 7.34 & 7.70 & 8.34 & 8.02 \\
\hline $\mathrm{Zn}_{2}+\mathrm{Mn}_{1}$ & 54.00 & 55.00 & $\mathbf{5 4 . 5 0}$ & 7.67 & 7.67 & 7.67 & 8.44 & 8.74 & 8.59 \\
\hline $\mathbf{Z n}_{2}+\mathbf{M n}_{2}$ & 56.33 & 57.33 & 56.83 & 8.00 & 8.33 & 8.17 & 8.80 & 9.16 & 8.98 \\
\hline Mean (A) & 49.59 & 50.67 & & 7.00 & 7.33 & & 7.70 & 8.07 & \\
\hline \multicolumn{10}{|l|}{$\operatorname{LSD}_{(0.05)}:$} \\
\hline (A) & & 1.01 & & & 0.19 & & & 0.31 & \\
\hline (B) & & 1.51 & & & 0.30 & & & 0.46 & \\
\hline (A) $\times$ (B) & & 2.00 & & & 0.37 & & & 0.61 & \\
\hline
\end{tabular}

$\mathrm{Zn}_{0}=$ water (control), $\mathrm{Zn}_{1}=100 \mathrm{ppm}$, and $\mathrm{Zn}_{2}=150 \mathrm{ppm}$.

$\mathrm{Mn}_{0}=$ water (control), $\mathrm{Mn}_{1}=100 \mathrm{ppm}$, and $\mathrm{Mn}_{2}=150 \mathrm{ppm}$.

Fayoum J. Agric. Res. \& Dev., Vol.19, No.2, July, 2005 
Mostafa M. Rady, et al.

Table 2b. Effect of soil N-application and foliar nutrition with $\mathrm{Zn}$ and $\mathrm{Mn}$ on vegetative growth characters of amaryllus plants during the two successive seasons 2003/2004 and 2004/2005.

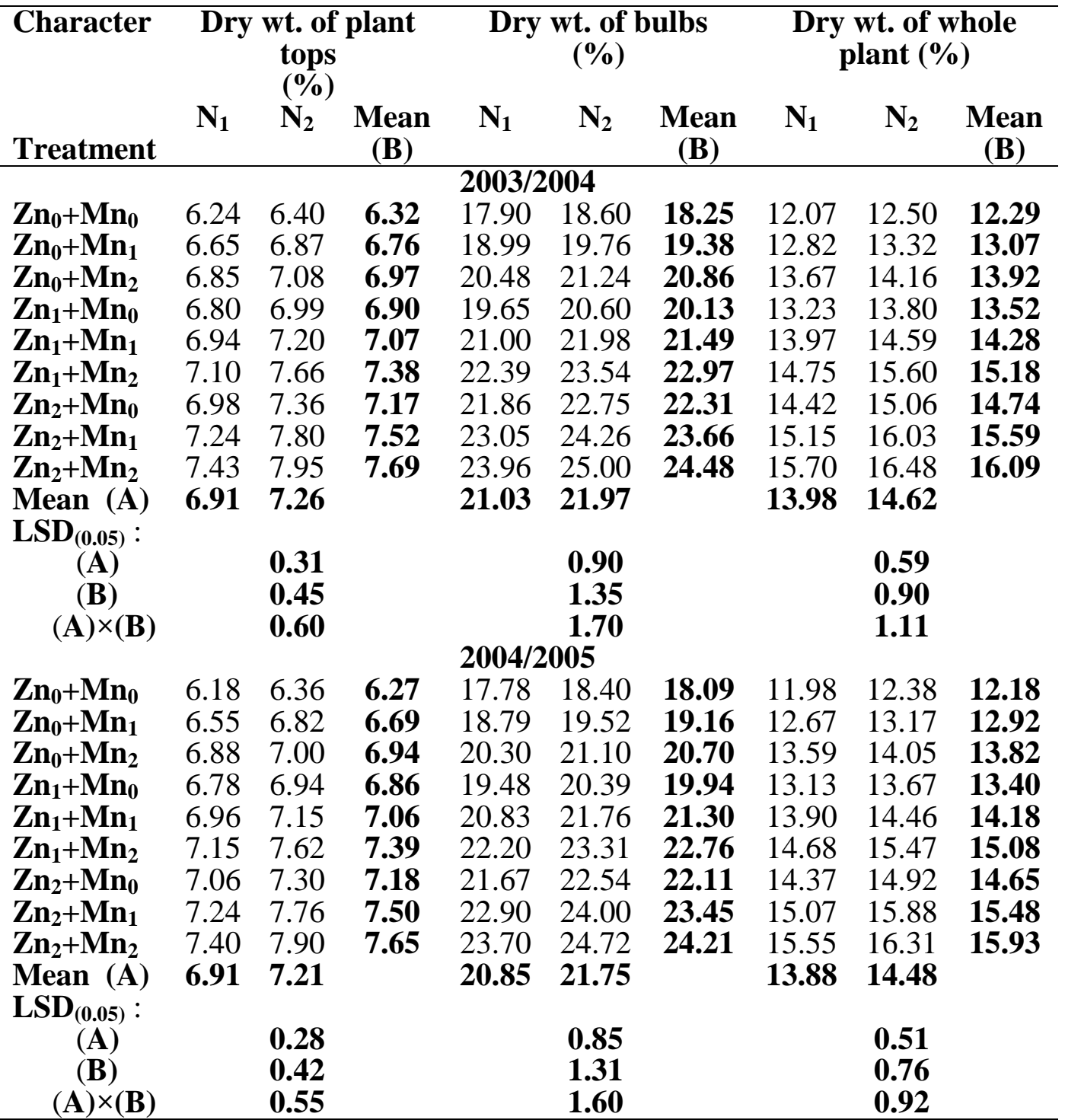

$\mathrm{Zn}_{0}=$ water (control), $\mathrm{Zn}_{1}=100 \mathrm{ppm}$, and $\mathrm{Zn}_{2}=150 \mathrm{ppm}$.

$\mathrm{Mn}_{0}=$ water (control), $\mathrm{Mn}_{1}=100 \mathrm{ppm}$, and $\mathrm{Mn}_{2}=150 \mathrm{ppm}$.

Fayoum J. Agric. Res. \& Dev., Vol.19, No.2, July, 2005 
EFFECT OF SOIL-N APPLICATION AND FOLIAR NUTRITION......85

Table 2c. Effect of soil $\mathrm{N}$-application and foliar nutrition with $\mathrm{Zn}$ and $\mathrm{Mn}$ on vegetative growth characters of amaryllus plants during the two successive seasons 2003/2004 and 2004/2005.

\begin{tabular}{|c|c|c|c|c|c|c|c|c|c|}
\hline \multirow{2}{*}{$\begin{array}{l}\text { Character } \\
\text { Treatment }\end{array}$} & \multicolumn{3}{|c|}{ No. of bulbs+bulblets/pot } & \multicolumn{3}{|c|}{$\begin{array}{c}\text { Bulb size } \\
\left(\mathrm{cm}^{3}\right)\end{array}$} & \multicolumn{3}{|c|}{$\begin{array}{c}\text { Fresh wt. of bulb } \\
\text { (g) }\end{array}$} \\
\hline & $\mathbf{N}_{1}$ & $\mathbf{N}_{2}$ & $\begin{array}{c}\text { Mean } \\
\text { (B) }\end{array}$ & $\mathbf{N}_{1}$ & $\mathbf{N}_{2}$ & $\begin{array}{c}\text { Mean } \\
\text { (B) }\end{array}$ & $\mathbf{N}_{1}$ & $\mathbf{N}_{2}$ & $\begin{array}{c}\text { Mean } \\
\text { (B) }\end{array}$ \\
\hline \multicolumn{10}{|c|}{$2003 / 2004$} \\
\hline $\mathbf{Z n}_{0}+\mathbf{M n} \mathbf{n}_{0}$ & 3.33 & 3.67 & 3.50 & 90.84 & 97.43 & 94.14 & 84.11 & 90.21 & 87.16 \\
\hline $\mathbf{Z n}_{0}+\mathbf{M n}_{1}$ & 3.67 & 4.00 & 3.84 & 96.96 & 102.96 & 99.96 & 89.36 & 94.89 & 92.13 \\
\hline $\mathrm{Zn}_{0}+\mathbf{M n}_{2}$ & 4.67 & 4.67 & 4.67 & 103.25 & 110.50 & 106.88 & 94.81 & 101.47 & 98.14 \\
\hline $\mathbf{Z n}_{1}+\mathbf{M n}_{0}$ & 4.00 & 4.67 & 4.34 & 99.50 & 106.86 & 103.18 & 91.20 & 97.95 & 94.58 \\
\hline $\mathbf{Z n}_{1}+\mathbf{M n} \mathbf{n}_{1}$ & 4.67 & 5.00 & 4.84 & 104.92 & 116.82 & 110.87 & 96.08 & 106.98 & 101.53 \\
\hline $\mathbf{Z n}_{1}+\mathbf{M n}_{2}$ & 5.33 & 5.67 & 5.50 & 140.78 & 143.14 & 141.96 & 128.68 & 130.65 & 129.67 \\
\hline $\mathbf{Z n}_{2}+\mathbf{M n}_{0}$ & 5.00 & 5.33 & 5.17 & 132.42 & 136.19 & 134.31 & 121.15 & 123.69 & 122.42 \\
\hline $\mathbf{Z n}_{2}+\mathbf{M n} \mathbf{n}_{1}$ & 5.67 & 6.33 & 6.00 & 149.32 & 153.89 & 151.61 & 136.12 & 140.28 & 138.20 \\
\hline $\mathrm{Zn}_{2}+\mathrm{Mn}_{2}$ & 6.33 & 6.67 & 6.50 & 162.96 & 167.86 & 165.41 & 148.15 & 152.60 & 150.38 \\
\hline Mean (A) & 4.74 & 5.11 & & 120.11 & 126.18 & & 109.96 & 115.41 & \\
\hline \multicolumn{10}{|l|}{$\operatorname{LSD}_{(0.05)}$ : } \\
\hline (A) & & 0.20 & & & 5.40 & & & 5.11 & \\
\hline (B) & & 0.30 & & & 8.10 & & & 7.65 & \\
\hline$(\mathbf{A}) \times(\mathbf{B})$ & & 0.38 & & & 9.80 & & & 9.21 & \\
\hline \multicolumn{10}{|c|}{$2004 / 2005$} \\
\hline $\mathbf{Z n}_{0}+\mathbf{M n}_{0}$ & 3.00 & 3.33 & 3.17 & 85.42 & 91.45 & 88.44 & 79.09 & 84.68 & 81.89 \\
\hline $\mathrm{Zn}_{0}+\mathbf{M n}_{1}$ & 3.33 & 3.67 & 3.50 & 91.81 & 97.41 & 94.61 & 84.62 & 89.78 & 87.20 \\
\hline $\mathrm{Zn}_{0}+\mathrm{Mn}_{2}$ & 4.00 & 4.33 & 4.17 & 108.50 & 112.62 & 110.56 & 99.82 & 103.61 & 101.72 \\
\hline $\mathbf{Z n}_{1}+\mathbf{M n}_{0}$ & 3.67 & 4.00 & 3.84 & 101.12 & 105.48 & 103.30 & 93.20 & 97.16 & 95.18 \\
\hline $\mathbf{Z n}_{1}+\mathbf{M n} \mathbf{n}_{1}$ & 4.67 & 4.67 & 4.67 & 119.92 & 125.48 & 122.70 & 110.02 & 115.12 & 112.57 \\
\hline $\mathbf{Z n}_{1}+\mathbf{M n} \mathbf{n}_{2}$ & 4.67 & 5.33 & 5.00 & 136.49 & 141.80 & 139.15 & 124.65 & 129.50 & 127.08 \\
\hline $\mathbf{Z n}_{2}+\mathbf{M n}_{0}$ & 4.67 & 5.00 & 4.84 & 127.12 & 132.80 & 129.96 & 116.30 & 121.50 & 118.90 \\
\hline $\mathbf{Z n}_{2}+\mathbf{M n}_{1}$ & 5.33 & 5.67 & 5.50 & 145.59 & 150.44 & 148.02 & 132.72 & 137.14 & 134.93 \\
\hline $\mathrm{Zn}_{2}+\mathrm{Mn}_{2}$ & 6.00 & 6.33 & 6.17 & 157.56 & 161.66 & 159.61 & 143.24 & 146.96 & 145.10 \\
\hline Mean (A) & 4.37 & 4.70 & & 119.28 & 124.35 & & 109.30 & 113.94 & \\
\hline \multicolumn{10}{|l|}{$\operatorname{LSD}_{(0.05)}$ : } \\
\hline (A) & & 0.29 & & & 5.00 & & & 4.60 & \\
\hline (B) & & 0.43 & & & 7.45 & & & 6.90 & \\
\hline$(\mathbf{A}) \times(\mathbf{B})$ & & 0.57 & & & 9.05 & & & 8.20 & \\
\hline
\end{tabular}

$\mathrm{Zn}_{0}=$ water (control), $\mathrm{Zn}_{1}=100 \mathrm{ppm}$, and $\mathrm{Zn}_{2}=150 \mathrm{ppm}$.

$\mathrm{Mn}_{0}=$ water (control), $\mathrm{Mn}_{1}=100 \mathrm{ppm}$, and $\mathrm{Mn}_{2}=150 \mathrm{ppm}$.

\section{Flowering traits:}

Data given in Tables ( $3 a$ and $3 b$ ) indicate that, increasing $N$ rate from 2 to $4 \mathrm{~g} \mathrm{NH}_{4} \mathrm{NO}_{3}$ /plant significantly promoted the number of flowers/floral stalk, floral stalk length, average of fresh weight/floral stalk and keeping quality. The obtained results are in harmony with those reported by Dahya et al. (1998), Chadha et al. (1999), Hameed and Sakar (1999), Ram et al. (1999) and Broschat and Moore (2001) on marigold plant and El-Gendy et al. (2001) on Ocimum basilicum. They concluded that nitrogen fertilization increases flowering traits.

Fayoum J. Agric. Res. \& Dev., Vol.19, No.2, July, 2005 
Mostafa M. Rady, et al.

Table 3a. Effect of soil N-application and foliar nutrition with $\mathrm{Zn}$ and $\mathrm{Mn}$ on flowering traits of amaryllus plants during the two successive seasons 2003/2004 and 2004/2005.

\begin{tabular}{|c|c|c|c|c|c|c|c|c|c|}
\hline \multirow{3}{*}{$\begin{array}{l}\text { Character } \\
\text { Treatment }\end{array}$} & \multicolumn{6}{|c|}{ Keeping quality from cutting time to } & \multirow{2}{*}{\multicolumn{3}{|c|}{$\begin{array}{l}\text { Mean of keeping } \\
\text { quality (vase life) } \\
\text { (day) }\end{array}$}} \\
\hline & \multicolumn{3}{|c|}{$\begin{array}{l}1^{\text {st }} \text { flower death on } \\
\text { floral stalk (day) }\end{array}$} & \multicolumn{3}{|c|}{$\begin{array}{l}\text { Last flower death on } \\
\text { floral stalk (day) }\end{array}$} & & & \\
\hline & $\mathbf{N}_{1}$ & $\mathbf{N}_{2}$ & $\begin{array}{l}\text { Mean } \\
\text { (B) }\end{array}$ & $\mathbf{N}_{1}$ & $\mathbf{N}_{2}$ & $\begin{array}{l}\text { Mean } \\
\text { (B) }\end{array}$ & $\mathbf{N}_{1}$ & $\mathbf{N}_{2}$ & $\begin{array}{c}\text { Mean } \\
\text { (B) }\end{array}$ \\
\hline \multicolumn{10}{|c|}{$2003 / 2004$} \\
\hline $\mathbf{Z n}_{0}+\mathbf{M n} \mathbf{n}_{0}$ & 5.67 & 6.00 & 5.84 & 8.33 & 8.67 & 8.50 & 7.00 & 7.34 & 7.17 \\
\hline $\mathbf{Z n}_{0}+\mathbf{M n}_{1}$ & 6.00 & 6.67 & 6.34 & 8.67 & 9.33 & 9.00 & 7.34 & 8.00 & 7.67 \\
\hline $\mathbf{Z n}_{0}+\mathbf{M n} \mathbf{n}_{2}$ & 6.67 & 7.33 & 7.00 & 9.00 & 9.67 & 9.34 & 7.84 & 8.50 & 8.17 \\
\hline $\mathbf{Z n}_{1}+\mathbf{M n} \mathbf{n}_{0}$ & 6.33 & 7.00 & 6.67 & 9.00 & 9.67 & 9.34 & 7.67 & 8.34 & 8.01 \\
\hline $\mathbf{Z n}_{1}+\mathbf{M n} \mathbf{n}_{1}$ & 7.00 & 7.67 & 7.34 & 9.33 & 9.67 & 9.50 & 8.17 & 8.67 & 8.42 \\
\hline $\mathbf{Z n}_{1}+\mathbf{M n} \mathbf{n}_{2}$ & 7.67 & 8.00 & 7.84 & 9.67 & 10.33 & 10.00 & 8.67 & 9.17 & 8.92 \\
\hline $\mathbf{Z n}_{2}+\mathbf{M n} \mathbf{n}_{0}$ & 7.67 & 8.00 & 7.84 & 9.67 & 10.00 & 9.84 & 8.67 & 9.00 & 8.84 \\
\hline $\mathbf{Z n}_{2}+\mathbf{M n} \mathbf{n}_{1}$ & 8.00 & 8.33 & 8.17 & 10.00 & 10.33 & 10.17 & 9.00 & 9.33 & 9.17 \\
\hline $\mathbf{Z} \mathbf{n}_{2}+\mathbf{M} n_{2}$ & 8.00 & 8.67 & 8.34 & 10.33 & 10.67 & 10.50 & 9.17 & 9.67 & 9.42 \\
\hline Mean (A) & 7.00 & 7.52 & & 9.33 & 9.82 & & 8.17 & 8.67 & \\
\hline \multicolumn{10}{|l|}{$\mathbf{L S D}_{(0.05)}:$} \\
\hline (A) & & 0.45 & & & 0.43 & & & 0.36 & \\
\hline (B) & & 0.68 & & & 0.65 & & & 0.54 & \\
\hline$(A) \times(B)$ & & 0.89 & & & 0.84 & & & 0.71 & \\
\hline \multicolumn{10}{|c|}{$2004 / 2005$} \\
\hline $\mathbf{Z n}_{0}+\mathbf{M n} \mathbf{n}_{0}$ & 5.33 & 5.67 & 5.50 & 8.67 & 9.33 & 9.00 & 7.00 & 7.50 & 7.25 \\
\hline $\mathbf{Z n}_{0}+\mathbf{M n} \mathbf{n}_{1}$ & 5.67 & 6.00 & 5.84 & 9.33 & 9.67 & 9.50 & 7.50 & 7.84 & 7.67 \\
\hline $\mathbf{Z} \mathbf{n}_{0}+\mathbf{M n} \mathbf{n}_{2}$ & 6.00 & 6.67 & 6.34 & 9.67 & 10.00 & 9.84 & 7.84 & 8.34 & 8.09 \\
\hline $\mathbf{Z} \mathbf{n}_{1}+\mathbf{M n} \mathbf{n}_{0}$ & 6.00 & 6.33 & 6.17 & 9.67 & 10.00 & 9.84 & 7.84 & 8.17 & 8.01 \\
\hline $\mathbf{Z n}_{1}+\mathbf{M} \mathbf{n}_{1}$ & 6.33 & 6.67 & 6.50 & 9.67 & 10.33 & 10.00 & 8.00 & 8.50 & 8.25 \\
\hline $\mathbf{Z} \mathbf{n}_{1}+\mathbf{M n} n_{2}$ & 6.67 & 7.00 & 6.84 & 9.67 & 10.67 & 10.17 & 8.17 & 8.84 & 8.51 \\
\hline $\mathbf{Z n}_{2}+\mathbf{M} \mathbf{n}_{0}$ & 6.67 & 7.00 & 6.84 & 9.67 & 10.67 & 10.17 & 8.17 & 8.84 & 8.51 \\
\hline $\mathbf{Z n}_{2}+\mathbf{M n} n_{1}$ & 7.00 & 7.33 & 7.17 & 10.33 & 11.00 & 10.67 & 8.67 & 9.17 & 8.92 \\
\hline $\mathbf{Z} \mathbf{n}_{2}+\mathbf{M} n_{2}$ & 7.67 & 7.67 & 7.67 & 10.33 & 11.00 & 10.67 & 9.00 & 9.34 & 9.17 \\
\hline Mean (A) & 6.37 & 6.70 & & 9.67 & 10.30 & & 8.02 & 8.50 & \\
\hline \multicolumn{10}{|l|}{$\mathbf{L S D}_{(0.05)}:$} \\
\hline (A) & & 0.31 & & & 0.50 & & & 0.42 & \\
\hline (B) & & 0.46 & & & 0.74 & & & 0.63 & \\
\hline$(\mathrm{A}) \times(\mathrm{B})$ & & 0.60 & & & 0.98 & & & 0.81 & \\
\hline
\end{tabular}

$\mathrm{Zn}_{0}=$ water (control), $\mathrm{Zn}_{1}=100 \mathrm{ppm}$, and $\mathrm{Zn}_{2}=150 \mathrm{ppm}$.

$\mathrm{Mn}_{0}=$ water (control), $\mathrm{Mn}_{1}=100 \mathrm{ppm}$, and $\mathrm{Mn}_{2}=150 \mathrm{ppm}$.

Regarding the infleunce of foliar spray of amaryllus plants with $\mathrm{Zn}$ and/or Mn, data indicate that the mixture of both $\mathrm{Zn}$ and $\mathrm{Mn}$ at a concentration of $150 \mathrm{ppm}$ for each significantly increased all the studied flowering trait parameters in both seasons (tables $3 \mathrm{a}$ and $3 \mathrm{~b}$ ). In this respect, Selim and Tantawy (1993) on gerbera, El-Deeb (1999) on Philodendron scandens, Refaat and Balbaa (2001) on lemongrass plants and Selim et al. (2001) on Calendula officinalis, mentioned that spraying plants with $\mathrm{Zn}$ or Mn increase flowering traits.

Fayoum J. Agric. Res. \& Dev., Vol.19, No.2, July, 2005 
EFFECT OF SOIL-N APPLICATION AND FOLIAR NUTRITION.....87

Table 3b. Effect of soil N-application and foliar nutrition with $\mathrm{Zn}$ and $\mathrm{Mn}$ on flowering traits of amaryllus plants during the two successive seasons 2003/2004 and 2004/2005.

\begin{tabular}{|c|c|c|c|c|c|c|c|c|c|}
\hline \multirow{2}{*}{$\begin{array}{l}\text { Character } \\
\text { Treatment }\end{array}$} & \multicolumn{3}{|c|}{$\begin{array}{l}\text { Length of floral } \\
\text { stalk (cm) }\end{array}$} & \multicolumn{3}{|c|}{$\begin{array}{l}\text { No. of flowers/ } \\
\text { floral stalk }\end{array}$} & \multicolumn{3}{|c|}{$\begin{array}{l}\text { Fresh wt. of flowers } \\
\text { /floral stalk (g) }\end{array}$} \\
\hline & $\mathbf{N}_{1}$ & $\mathbf{N}_{2}$ & $\begin{array}{c}\text { Mean } \\
(\mathrm{B})\end{array}$ & $\mathbf{N}_{1}$ & $\mathbf{N}_{2}$ & $\begin{array}{c}\text { Mean } \\
(\mathrm{B})\end{array}$ & $\mathbf{N}_{1}$ & $\mathbf{N}_{2}$ & $\begin{array}{c}\text { Mean } \\
\text { (B) }\end{array}$ \\
\hline & \multicolumn{9}{|c|}{$2003 / 2004$} \\
\hline $\mathbf{Z n}_{0}+\mathbf{M n}_{0}$ & 23.67 & 26.33 & 25.00 & 2.00 & 2.33 & 2.17 & 19.20 & 22.37 & 20.79 \\
\hline $\mathbf{Z n}_{0}+\mathbf{M n}_{1}$ & 26.33 & 30.00 & 28.17 & 2.33 & 2.67 & 2.50 & 22.34 & 25.63 & 23.99 \\
\hline $\mathbf{Z n}_{0}+\mathbf{M n}_{2}$ & 30.67 & 35.33 & 33.00 & 2.67 & 3.00 & 2.84 & 25.77 & 28.95 & 27.36 \\
\hline $\mathbf{Z n}_{1}+\mathbf{M n}_{0}$ & 29.33 & 33.67 & 31.50 & 2.67 & 3.00 & 2.84 & 25.75 & 28.90 & 27.33 \\
\hline $\mathbf{Z} \mathbf{n}_{1}+\mathbf{M n} \mathbf{n}_{1}$ & 32.67 & 38.00 & 35.34 & 2.67 & 3.33 & 3.00 & 25.90 & 32.30 & 29.10 \\
\hline $\mathbf{Z n}_{1}+\mathbf{M n} \mathbf{n}_{2}$ & 37.33 & 43.33 & 40.33 & 3.00 & 3.67 & 3.34 & 29.10 & 35.60 & 32.35 \\
\hline $\mathbf{Z n}_{2}+\mathbf{M n}_{0}$ & 36.67 & 41.67 & 39.17 & 3.00 & 3.67 & 3.34 & 29.25 & 35.78 & 32.52 \\
\hline $\mathbf{Z n}_{2}+\mathbf{M n}_{1}$ & 41.67 & 46.67 & 44.17 & 3.67 & 3.67 & 3.67 & 35.96 & 36.00 & 35.98 \\
\hline $\mathbf{Z} \mathbf{n}_{2}+\mathbf{M n} \mathbf{n}_{2}$ & 44.33 & 50.00 & 47.17 & 4.00 & 4.00 & 4.00 & 39.20 & 39.36 & 39.28 \\
\hline Mean (A) & 33.63 & 38.33 & & 2.89 & 3.26 & & 28.05 & 31.65 & \\
\hline \multirow{5}{*}{$\begin{array}{c}\operatorname{LSD}_{(\mathbf{0 . 0 5})}: \\
(\mathbf{A}) \\
(\mathbf{B}) \\
(\mathbf{A}) \times(\mathbf{B})\end{array}$} & & & & & & & & & \\
\hline & & 2.60 & & & 0.21 & & & 2.45 & \\
\hline & & 3.88 & & & 0.31 & & & 3.60 & \\
\hline & & 5.11 & & & 0.40 & & & 4.80 & \\
\hline & \multicolumn{9}{|c|}{$2004 / 2005$} \\
\hline $\mathbf{Z n}_{0}+\mathbf{M n}_{0}$ & 25.00 & 27.33 & 26.17 & 2.00 & 2.00 & 2.00 & 19.04 & 19.36 & 19.20 \\
\hline $\mathbf{Z} \mathbf{n}_{0}+\mathbf{M n} \mathbf{n}_{1}$ & 28.33 & 30.67 & 29.50 & 2.33 & 2.33 & 2.33 & 22.25 & 22.72 & 22.49 \\
\hline $\mathbf{Z n}_{0}+\mathbf{M n}_{2}$ & 32.00 & 34.67 & 33.34 & 2.33 & 2.67 & 2.50 & 22.31 & 26.04 & 24.18 \\
\hline $\mathbf{Z n}_{1}+\mathbf{M n}_{0}$ & 30.67 & 33.67 & 32.17 & 2.33 & 2.67 & 2.50 & 22.31 & 25.96 & 24.14 \\
\hline $\mathbf{Z} \mathbf{n}_{1}+\mathbf{M n} \mathbf{n}_{1}$ & 34.00 & 36.00 & 35.00 & 2.67 & 3.00 & 2.84 & 25.63 & 29.40 & 27.52 \\
\hline $\mathbf{Z n}_{1}+\mathbf{M n} \mathbf{n}_{2}$ & 39.00 & 39.67 & 39.34 & 3.00 & 3.67 & 3.34 & 28.95 & 36.15 & 32.55 \\
\hline $\mathrm{Zn}_{2}+\mathrm{Mn}_{0}$ & 37.33 & 39.00 & 38.17 & 3.00 & 3.33 & 3.17 & 28.80 & 32.64 & 30.72 \\
\hline $\mathrm{Zn}_{2}+\mathrm{Mn}_{1}$ & 41.67 & 43.00 & 42.34 & 3.33 & 3.67 & $\mathbf{3 . 5 0}$ & 32.30 & 36.26 & 34.28 \\
\hline $\mathbf{Z n}_{2}+\mathbf{M n} \mathbf{n}_{2}$ & 43.67 & 46.33 & 45.00 & 3.67 & 4.00 & 3.84 & 35.96 & 39.20 & 37.58 \\
\hline Mean (A) & 34.63 & 36.70 & & 2.74 & 3.04 & & 26.39 & 29.75 & \\
\hline \multirow{2}{*}{\multicolumn{10}{|c|}{$\underset{(\mathbf{A S D})}{\operatorname{LSD}_{(0.05)}}$}} \\
\hline & & N.S. & & & & & & & \\
\hline & & 3.55 & & & 0.29 & & & 3.96 & \\
\hline$(\mathbf{A}) \times(\mathbf{B})$ & & 4.70 & & & 0.39 & & & 5.30 & \\
\hline
\end{tabular}

$\mathrm{Zn}_{0}=$ water (control), $\mathrm{Zn}_{1}=100 \mathrm{ppm}$, and $\mathrm{Zn}_{2}=150 \mathrm{ppm}$.

$\mathrm{Mn}_{0}=$ water (control), $\mathrm{Mn}_{1}=100 \mathrm{ppm}$, and $\mathrm{Mn}_{2}=150 \mathrm{ppm}$.

The interacting effects of $\mathrm{N}$ levels with $\mathrm{Zn}$ or $\mathrm{Mn}$ and their mixtures at any concentration on flowering traits varied between the two seasons. Comparison among the different treatments clearly indicated that, fertilization with $4 \mathrm{~g}$ ammonium nitrate/pot in combination with the mixture of $\mathrm{Zn}$ and $\mathrm{Mn}$ foliar spray at a concentration of $150 \mathrm{ppm}$ for each resulted in the best mean values for all flowering parameters.

Fayoum J. Agric. Res. \& Dev., Vol.19, No.2, July, 2005 


\section{Chemical composition of plant tops and flowers: 1.Pigments and total carbohydrates:}

Data presented in Tables (4 and 5) show that, increasing $\mathrm{N}$ rate from 2 to $4 \mathrm{~g} \mathrm{NH}_{4} \mathrm{NO}_{3}$ /plant significantly increased chlorophyll a and $\mathrm{b}$, total carotenoids and total carbohydrates in leaves, and anthocyanin in flowers either at picking time or 5 days later. This trend was similar in both seasons. In this respect, Yadav et al. (1999) reported that, total leaf chlorophyll in African marigold increases consistently as a result of increasing $\mathrm{N}$-application up to $180 \mathrm{ppm}$.

Regarding the effect of foliar spray with $\mathrm{Zn}$ or $\mathrm{Mn}$ and their mixtures, data in Tables (4 and 5) reveal that, $\mathrm{Zn}$ and $\mathrm{Mn}$ at $150 \mathrm{ppm}$ concentration produced the highest values of chlorophylls ( $a$ and $b$ ), carotenoids and total carbohydrates in leaves and anthocyanin content of flowers.

Generally, spraying the plants with $\mathrm{Zn}$ alone at any concentration gave the best results compared with $\mathrm{Mn}$. These results may be due to the basic function of zinc in plant which is related to its role in the metabolism of carbohydrates (Price $\boldsymbol{e t}$ al., 1972). On the other hand, plants treated with the mixtures of $\mathrm{Zn}$ and $\mathrm{Mn}$ at different concentrations recorded the best values. These results may be attributed to their combined enhancing effect on most metabolic processes such as carbohydrates and chlorophyll formation (Price $\boldsymbol{e t}$ al., 1972, Bidwell, 1980 and Mohr and Schopfer, 1995). The results are in harmony with findings of El-Deeb (1999) on Philodendron scandens and Selim et al. (2001) on Calendula officinalis who mentioned that, $\mathrm{Zn}$ and $\mathrm{Mn}$ increased chlorophylls ( $\mathrm{a}$ and $\mathrm{b}$ ) and carotenoids. While Refaat and Balbaa (2001) on lemongrass revealed that, $\mathrm{Zn}$ and $\mathrm{Mn}$ treated-plants showed lower values of total carbohydrates comparable to untreated plants.

The interaction between $\mathrm{N}$-fertilization and $\mathrm{Zn}$ or $\mathrm{Mn}$ and their mixtures as tabulated in Tables (4 and 5) exhibit that, determinations fluctuated significantly or not. The highest values of chlorophylls a and $b$, total carotenoids, anthocyanin and total carbohydrates were associated with the treatment $4 \mathrm{~g}$ ammonium nitrate/plant in combination with foliar spray of the mixture $(\mathrm{Zn}+\mathrm{Mn})$ at a concentreation of $150 \mathrm{ppm}$ for each.

2. Nitrogen, phosphorus and potassium:

Data listed in Table (6) indicate that, the percentages of $\mathrm{N}$ and $\mathrm{P}$ in leaves significantly increased as a result of treating plants with $4 \mathrm{~g}$ ammonium nitrate/plant in both seasons. In this respect, Yadav et al. (1999) reported that, total nitrogen content of African marigold consistently increased with $\mathrm{N}$ application up to $180 \mathrm{ppm}$. While for $\mathrm{K}$, the highest value of $\mathrm{K}$ content was determined in plants treated with $2 \mathrm{~g}$ ammonium nitrate/plant.

Foliar spray with zinc and manganese at the concentration of $150 \mathrm{ppm}$ for each increased total nitrogen and potassium concentrations in leaves followed by the mixtures of $\mathrm{Zn}(150 \mathrm{ppm})$ with $\mathrm{Mn}(100 \mathrm{ppm})$ then $\mathrm{Zn}(100$ $\mathrm{ppm}$ ) with $\mathrm{Mn}(150 \mathrm{ppm})$. Generally, all combinations of $\mathrm{Zn}$ and $\mathrm{Mn}$ concentrations increased the values of $\mathrm{N}$ and $\mathrm{K}$ concentrations. These results may be due to the role of $\mathrm{Mn}$ as essential in the final step of nitrate reduction to ammonia (Amberger, 1978). On the other side, the basic function of $\mathrm{Zn}$ in plant is related to its role in the metabolism of proteins (Price et al., 1972). It is also necessary for RNA and protein synthesis (Vallee and Walker, 1976). The highest percentage of $\mathrm{P}$ was determined in plants treated with $\mathrm{Mn}$ at 150

Fayoum J. Agric. Res. \& Dev., Vol.19, No.2, July, 2005 
EFFECT OF SOIL-N APPLICATION AND FOLIAR NUTRITION.....899 or $100 \mathrm{ppm}$, respectively, followed by the combination of $\mathrm{Zn}(100 \mathrm{ppm})$ with $\mathrm{Mn}(150 \mathrm{ppm})$ then $\mathrm{Zn}$ with $\mathrm{Mn}$ at the concentration of $150 \mathrm{ppm}$ for each.

Table 4. Effect of soil $\mathrm{N}$-application and foliar nutrition with $\mathrm{Zn}$ and $\mathrm{Mn}$ on chlorophylls and carotenoids (in leaves) of amaryllus plants during the two successive seasons 2003/2004 and 2004/2005.

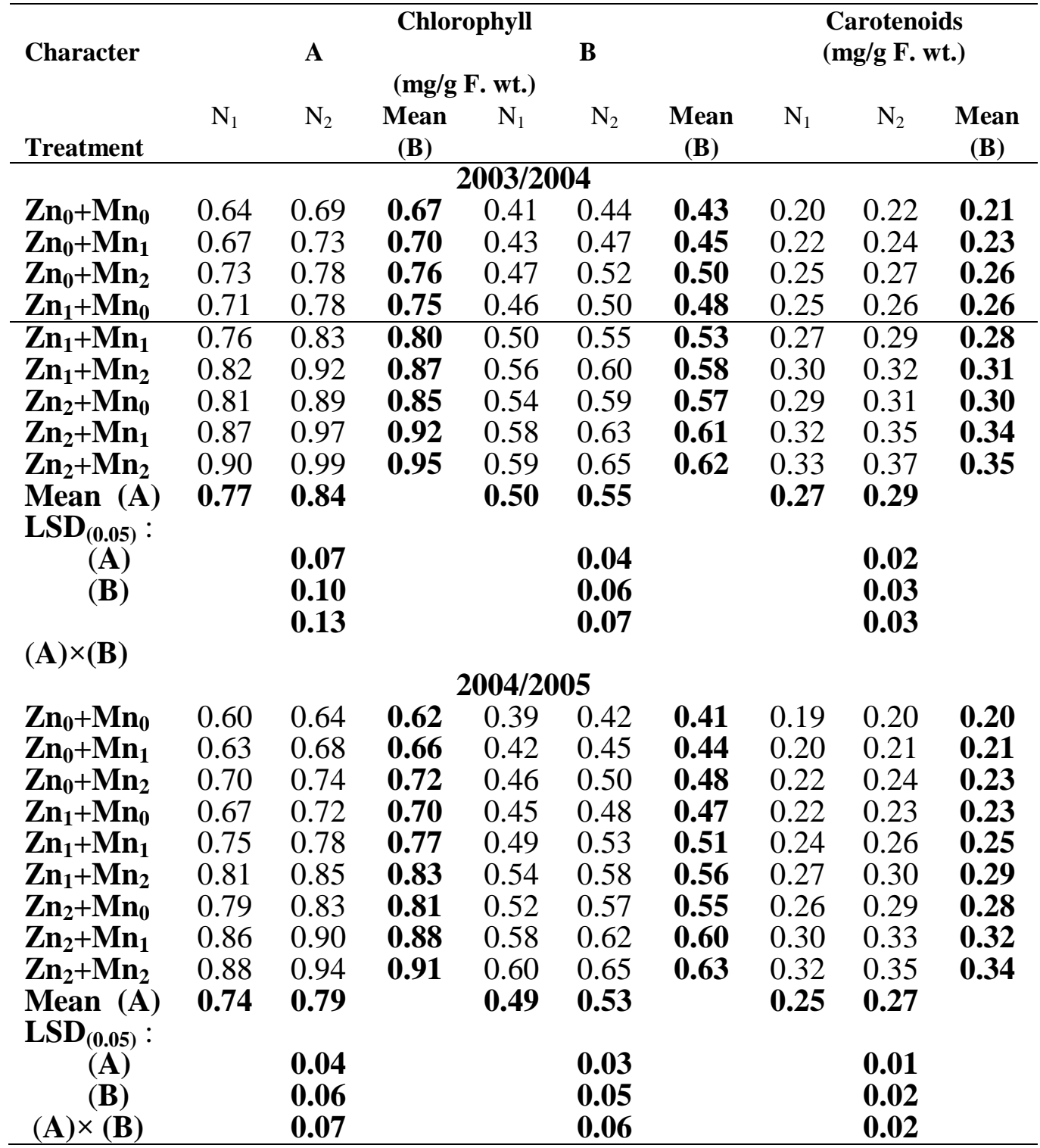

$\mathrm{Zn}_{0}=$ water (control), $\mathrm{Zn}_{1}=100 \mathrm{ppm}$, and $\mathrm{Zn}_{2}=150 \mathrm{ppm}$.

$\mathrm{Mn}_{0}=$ water (control), $\mathrm{Mn}_{1}=100 \mathrm{ppm}$, and $\mathrm{Mn}_{2}=150 \mathrm{ppm}$.

Fayoum J. Agric. Res. \& Dev., Vol.19, No.2, July, 2005 
Table 5. Effect of soil N-application and foliar nutrition with $\mathrm{Zn}$ and $\mathrm{Mn}$ on anthocyanin (in flowers) and carbohydrates (in leaves) of amaryllus plants during the two successive seasons 2003/2004 and 2004/2005.

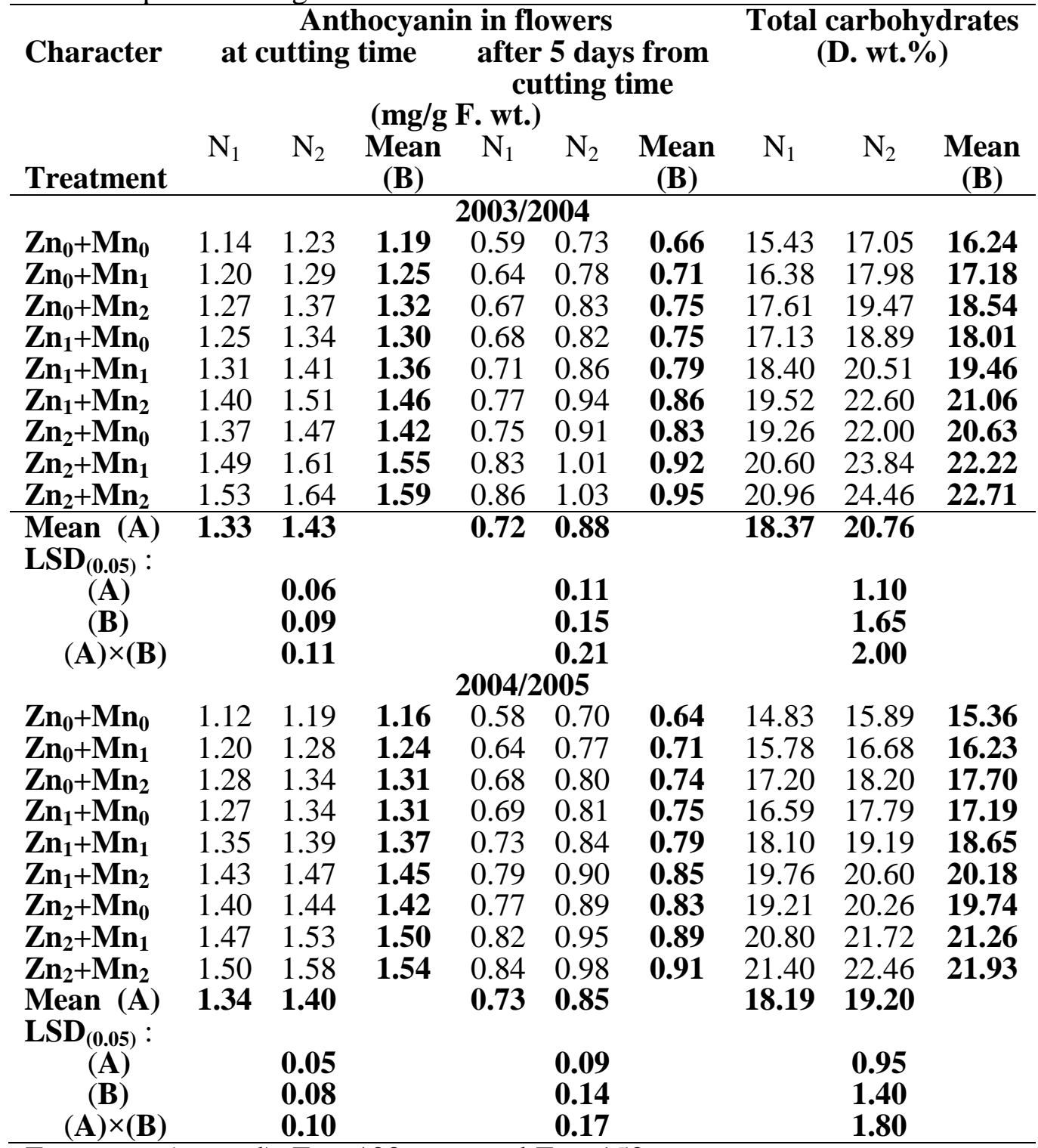

$\mathrm{Zn}_{0}=$ water (control), $\mathrm{Zn}_{1}=100 \mathrm{ppm}$, and $\mathrm{Zn}_{2}=150 \mathrm{ppm}$.

$\mathrm{Mn}_{0}=$ water (control), $\mathrm{Mn}_{1}=100 \mathrm{ppm}$, and $\mathrm{Mn}_{2}=150 \mathrm{ppm}$.

Concerning the combined effects of $\mathrm{N}$ levels with $\mathrm{Zn}$ and $\mathrm{Mn}$ on nitrogen accumulation in amaryllus leaves, data presented in Table (6) show that, fertilization with $4 \mathrm{~g}$ ammonium nitrate/plant in combination with $\mathrm{Zn}$ and $\mathrm{Mn}$ at the concentration of $150 \mathrm{ppm}$ for each resulted in the highest accumulation of nitrogen, followed by $4 \mathrm{~g} \mathrm{~N}$-fertilizer/pot $+\mathrm{Zn}$ and $\mathrm{Mn}$ at the concentrations of 150 and $100 \mathrm{ppm}$, respectively. Phosphorus accumulation in

Fayoum J. Agric. Res. \& Dev., Vol.19, No.2, July, 2005 
EFFECT OF SOIL-N APPLICATION AND FOLIAR NUTRITION......91 amaryllus leaves was highest in plants were fertilized with 2 or $4 \mathrm{~g}$ ammonium nitrate combined with $\mathrm{Mn}$ at $150 \mathrm{ppm} / \mathrm{plant}$, orderly.

Regarding the accumulation of $\mathrm{K}$ in leaves of amaryllus, the highest values were achieved when plants were fertilized with $2 \mathrm{~g} \quad \mathrm{NH}_{4} \mathrm{NO}_{3} /$ pot combined with the mixture of $\mathrm{Zn}$ and $\mathrm{Mn}$ at $150 \mathrm{ppm}$ for each, followed by 2 $\mathrm{g} /$ plant ammonium nitrate plus $\mathrm{Zn}$ and $\mathrm{Mn}$ at 150 and $100 \mathrm{ppm}$, orderly. These results were true in both seasons.

\section{Zinc, manganese and iron:}

\section{a. Zinc:}

Data in Table (7) show that, fertilizing plants with the lower $\mathrm{N}$ rate $(2$ $\mathrm{g} / \mathrm{plant}$ ) significantly increased the concentration of $\mathrm{Zn}$ in amaryllus leaves than the higher rate $(4 \mathrm{~g} / \mathrm{plant})$ in both seasons of study.

With regard to the combinal effect of $\mathrm{Zn}$ and $\mathrm{Mn}$, data in Table (7) clearly show that, the highest values of the determined $\mathrm{Zn}$ in amaryllus plant were recorded in plants sprayed with $\mathrm{Zn}$ at the concentration of $150 \mathrm{ppm}$ without Mn followed by the same concentration of $\mathrm{Zn}(150 \mathrm{ppm})$ mixed with $\mathrm{Mn}$ at the concentration of $100 \mathrm{ppm}$.

The interaction between $\mathrm{N}, \mathrm{Zn}$ and $\mathrm{Mn}$ show significant effect on $\mathrm{Zn}$ concentration in amaryllus plants. Data show that, fertilization with $2 \mathrm{~g}$ $\mathrm{NH}_{4} \mathrm{NO}_{3} /$ plant and $\mathrm{Zn}$ at the concentration of $150 \mathrm{ppm}$ resulted in significant increase in $\mathrm{Zn}$ concentration in plant leaves followed by plants fertilized with $2 \mathrm{~g} \mathrm{NH}_{4} \mathrm{NO}_{3} /$ plant and sprayed with $\mathrm{Zn}$ and $\mathrm{Mn}$ at 150 and $100 \mathrm{ppm}$, orderly.

\section{b. Manganese:}

Data presented in Table (7) clarify that, neither the $2 \mathrm{~g}$ ammonium nitrate/plant nor $4 \mathrm{~g} / \mathrm{plant}$ reflected any effect on $\mathrm{Mn}$ concentration in amaryllus leaves in both seasons.

Concerning the effect of $\mathrm{Zn}$ and Mn combinations, data clearly show that treating amaryllus plants with only $\mathrm{Mn}$ at the higher rate $(150 \mathrm{ppm})$ or with the same concentration of $\mathrm{Mn}$ and $\mathrm{Zn}$ resulted in the highest concentrations of $\mathrm{Mn}$ in amaryllus leaves, orderly in the two seasons of study.

The combinations of $\mathrm{N}, \mathrm{Zn}$ and Mn (Table, 7) clarify that, the highest Mn accumulation in amaryllus leaves was determined in plants fertilized with 2 or $4 \mathrm{~g} \mathrm{NH}_{4} \mathrm{NO}_{3} /$ plant and sprayed with the highest rate of $\mathrm{Mn}(150 \mathrm{ppm})$ in both seasons.

\section{c. Iron:}

Data presented in Table (7) show that, N-fertilization at $2 \mathrm{~g}$ or $4 \mathrm{~g}$ $\mathrm{NH}_{4} \mathrm{NO}_{3}$ /plant had no significant effect on the concentration of $\mathrm{Fe}$ in amaryllus plant in the both seasons of study.

Foliar spray with $\mathrm{Zn}$ at the concentration of $150 \mathrm{ppm}$ alone or mixed with $\mathrm{Mn}$ at the concentration of $100 \mathrm{ppm}$ resulted in the highest accumulation of $\mathrm{Fe}$ in leaves of amaryllus. These results are in agreement with the findings of Devlin and Witham (1985) who mentioned that $\mathrm{Zn}$ participates in different physiological functions or processes inside the plant including the processes of mineral uptake by plant roots and translocation inside the plant.

Regarding the combined effect of $\mathrm{N}$-fertilization, $\mathrm{Zn}$ and $\mathrm{Mn}$ foliar application, tabulated data in Table (7) clearly show that, the highest accumulation of $\mathrm{Fe}$ in amaryllus leaves were obtained when plants were fertilized with 2 or $4 \mathrm{~g}$ ammonium nitrate/plant and sprayed with $\mathrm{Zn}$ at the concentration of $150 \mathrm{ppm}$ in both seasons.

Fayoum J. Agric. Res. \& Dev., Vol.19, No.2, July, 2005 
Mostafa M. Rady, et al.

From all the above mentioned results of the present study, it could be concluded that the application of $\mathrm{Zn}$ and $\mathrm{Mn}$ as foliar sprays at a concentration of $150 \mathrm{ppm}$ for both in combination with soil-N application at the rate $4 \mathrm{~g} \mathrm{NH}_{4} \mathrm{NO}_{3}$ /plant produce a good keeping quality (long vase life) of flowering amaryllus plants.

Table 6. Effect of soil N-application and foliar nutrition with $\mathrm{Zn}$ and $\mathrm{Mn}$ on macroelements $(\mathrm{N}, \mathrm{P}$ and $\mathrm{K})$ in leaves of amaryllus plants during the two successive seasons 2003/2004 and 2004/2005.

\begin{tabular}{|c|c|c|c|c|c|c|c|c|c|}
\hline \multirow{2}{*}{$\begin{array}{l}\text { Character } \\
\text { Treatment }\end{array}$} & \multicolumn{3}{|c|}{$\mathbf{N}$} & \multicolumn{3}{|c|}{$\begin{array}{c}\mathbf{P} \\
(\% \text { D.wt. })\end{array}$} & \multicolumn{3}{|c|}{$\mathbf{K}$} \\
\hline & $\mathrm{N}_{1}$ & $\mathrm{~N}_{2}$ & $\begin{array}{c}\text { Mean } \\
\text { (B) }\end{array}$ & $\mathrm{N}_{1}$ & $\mathrm{~N}_{2}$ & $\begin{array}{c}\text { Mean } \\
\text { (B) }\end{array}$ & $\mathrm{N}_{1}$ & $\mathrm{~N}_{2}$ & $\begin{array}{c}\text { Mean } \\
\text { (B) }\end{array}$ \\
\hline \multicolumn{10}{|l|}{$2003 / 2004$} \\
\hline $\mathbf{Z n}_{0}+\mathbf{M n}_{0}$ & 1.96 & 2.17 & 2.07 & 0.31 & 0.32 & 0.32 & 2.61 & 2.32 & 2.47 \\
\hline $\mathrm{Zn}_{0}+\mathrm{Mn}_{1}$ & 2.06 & 2.30 & 2.18 & 0.33 & 0.34 & 0.34 & 2.80 & 2.45 & 2.63 \\
\hline $\mathrm{Zn}_{0}+\mathrm{Mn}_{2}$ & 2.18 & 2.41 & 2.30 & 0.34 & 0.35 & 0.35 & 2.95 & 2.61 & 2.78 \\
\hline $\mathrm{Zn}_{1}+\mathrm{Mn}_{0}$ & 2.12 & 2.36 & 2.24 & 0.31 & 0.31 & 0.31 & 2.78 & 2.46 & 2.62 \\
\hline $\mathbf{Z n}_{1}+\mathbf{M n}_{1}$ & 2.22 & 2.48 & 2.35 & 0.31 & 0.32 & 0.32 & 2.98 & 2.68 & 2.83 \\
\hline $\mathrm{Zn}_{1}+\mathbf{M n}_{2}$ & 2.34 & 2.61 & 2.48 & 0.32 & 0.33 & 0.33 & 3.11 & 2.73 & 2.92 \\
\hline $\mathrm{Zn}_{2}+\mathbf{M n}_{0}$ & 2.30 & 2.56 & 2.43 & 0.30 & 0.31 & 0.31 & 3.07 & 2.70 & 2.89 \\
\hline $\mathbf{Z n}_{2}+\mathbf{M n}_{1}$ & 2.39 & 2.69 & 2.54 & 0.31 & 0.31 & 0.31 & 3.14 & 2.81 & 2.98 \\
\hline $\mathbf{Z n}_{2}+\mathbf{M n}_{2}$ & 2.50 & 2.83 & 2.67 & 0.31 & 0.32 & 0.32 & 3.24 & 2.90 & $\mathbf{3 . 0 7}$ \\
\hline Mean (A) & 2.23 & 2.49 & & 0.32 & 0.32 & & 2.96 & 2.63 & \\
\hline \multicolumn{10}{|l|}{$\operatorname{LSD}_{(0.05)}:$} \\
\hline (A) & & 0.21 & & & N.S. & & & 0.20 & \\
\hline (B) & & 0.30 & & & 0.01 & & & 0.30 & \\
\hline$(\mathbf{A}) \times(\mathbf{B})$ & & 0.39 & & & 0.02 & & & 0.38 & \\
\hline \multicolumn{10}{|l|}{$2004 / 2005$} \\
\hline $\mathbf{Z n}_{0}+\mathbf{M n}_{0}$ & 2.01 & 2.15 & 2.08 & 0.33 & 0.34 & 0.34 & 2.53 & 2.27 & 2.40 \\
\hline $\mathrm{Zn}_{0}+\mathbf{M n}_{1}$ & 2.12 & 2.26 & 2.19 & 0.35 & 0.36 & 0.36 & 2.66 & 2.41 & 2.54 \\
\hline $\mathrm{Zn}_{0}+\mathrm{Mn}_{2}$ & 2.25 & 2.40 & 2.33 & 0.36 & 0.37 & 0.37 & 2.78 & 2.54 & 2.66 \\
\hline $\mathbf{Z n}_{1}+\mathbf{M n}_{0}$ & 2.18 & 2.34 & 2.26 & 0.31 & 0.32 & 0.32 & 2.68 & 2.42 & 2.55 \\
\hline $\mathbf{Z n}_{1}+\mathbf{M n}_{1}$ & 2.29 & 2.44 & 2.37 & 0.33 & 0.34 & 0.34 & 2.90 & 2.65 & 2.78 \\
\hline $\mathrm{Zn}_{1}+\mathrm{Mn}_{2}$ & 2.41 & 2.56 & 2.49 & 0.34 & 0.35 & 0.35 & 2.99 & 2.75 & 2.87 \\
\hline $\mathrm{Zn}_{2}+\mathbf{M n}_{0}$ & 2.35 & 2.54 & 2.45 & 0.31 & 0.32 & 0.32 & 2.91 & 2.65 & 2.78 \\
\hline $\mathrm{Zn}_{2}+\mathbf{M n}_{1}$ & 2.47 & 2.66 & 2.57 & 0.32 & 0.33 & 0.33 & 3.05 & 2.81 & 2.93 \\
\hline $\mathbf{Z} \mathbf{n}_{2}+\mathbf{M n}_{2}$ & 2.60 & 2.82 & 2.71 & 0.33 & 0.34 & 0.34 & 3.19 & 2.94 & 3.07 \\
\hline Mean (A) & 2.30 & 2.46 & & $\mathbf{0 . 3 3}$ & 0.34 & & 2.85 & 2.60 & \\
\hline \multicolumn{10}{|l|}{$\mathbf{L S D}_{(0.05)}:$} \\
\hline (A) & & 0.14 & & & 0.01 & & & 0.22 & \\
\hline (B) & & 0.21 & & & 0.01 & & & $\mathbf{0 . 3 3}$ & \\
\hline$(\mathbf{A}) \times(\mathbf{B})$ & & 0.27 & & & 0.02 & & & 0.42 & \\
\hline
\end{tabular}

$\mathrm{Zn}_{0}=$ water (control), $\mathrm{Zn}_{1}=100 \mathrm{ppm}$, and $\mathrm{Zn}_{2}=150 \mathrm{ppm}$.

$\mathrm{Mn}_{0}=$ water (control), $\mathrm{Mn}_{1}=100 \mathrm{ppm}$, and $\mathrm{Mn}_{2}=150 \mathrm{ppm}$.

Fayoum J. Agric. Res. \& Dev., Vol.19, No.2, July, 2005 
EFFECT OF SOIL-N APPLICATION AND FOLIAR NUTRITION......93

Table 7. Effect of soil $\mathrm{N}$-application and foliar nutrition with $\mathrm{Zn}$ and $\mathrm{Mn}$ on microelements ( $\mathrm{Zn}, \mathrm{Mn}$ and $\mathrm{Fe}$ ) in leaves of amaryllus plants during the two successive seasons 2003/2004 and 2004/2005.

\begin{tabular}{|c|c|c|c|c|c|c|c|c|c|}
\hline \multirow{2}{*}{$\begin{array}{l}\text { Character } \\
\text { Treatment }\end{array}$} & \multicolumn{3}{|c|}{ Zn } & \multicolumn{3}{|c|}{$\begin{array}{c}\text { Mn } \\
(\mu \mathrm{g} / \mathrm{g} \text { D. wt.) }\end{array}$} & \multicolumn{3}{|c|}{$\mathbf{F e}$} \\
\hline & $\mathrm{N}_{1}$ & $\mathrm{~N}_{2}$ & $\begin{array}{c}\text { Mean } \\
\text { (B) }\end{array}$ & $\mathrm{N}_{1}$ & $\mathrm{~N}_{2}$ & $\begin{array}{c}\text { Mean } \\
\text { (B) }\end{array}$ & $\mathrm{N}_{1}$ & $\mathrm{~N}_{2}$ & $\begin{array}{c}\text { Mean } \\
\text { (B) }\end{array}$ \\
\hline \multicolumn{10}{|c|}{$2003 / 2004$} \\
\hline $\mathbf{Z n}_{0}+\mathbf{M} \mathbf{n}_{0}$ & 78.20 & 71.96 & 75.08 & 121.46 & 121.68 & 121.57 & 210.26 & 212.00 & 211.13 \\
\hline $\mathrm{Zn}_{0}+\mathrm{Mn}_{1}$ & 77.62 & 71.50 & 74.56 & 128.63 & 127.95 & 128.29 & 210.61 & 210.96 & 210.79 \\
\hline $\mathbf{Z n}_{0}+\mathbf{M n}_{2}$ & 77.20 & 70.95 & 74.08 & 137.48 & 136.96 & 137.22 & 208.11 & 209.03 & 208.57 \\
\hline $\mathbf{Z} \mathbf{n}_{1}+\mathbf{M n}_{0}$ & 86.24 & 77.12 & 81.68 & 119.98 & 120.21 & 120.10 & 214.40 & 215.16 & 214.78 \\
\hline $\mathbf{Z} \mathbf{n}_{1}+\mathbf{M n}_{1}$ & 85.66 & 76.67 & 81.17 & 126.02 & 126.26 & 126.14 & 210.00 & 211.85 & 210.93 \\
\hline $\mathbf{Z} n_{1}+M n_{2}$ & 85.21 & 76.30 & 80.76 & 131.99 & 132.60 & 132.30 & 209.12 & 209.88 & 209.50 \\
\hline $\mathbf{Z n}_{2}+\mathbf{M} \mathbf{n}_{0}$ & 93.44 & 83.80 & 88.62 & 119.10 & 118.88 & 118.99 & 219.46 & 218.99 & 219.23 \\
\hline $\mathbf{Z n}_{2}+\mathbf{M n} \mathbf{n}_{1}$ & 92.68 & 83.42 & 88.05 & 125.62 & 125.41 & 125.52 & 216.70 & 217.00 & 216.85 \\
\hline $\mathbf{Z} \mathbf{n}_{2}+\mathbf{M n} \mathbf{n}_{2}$ & 92.20 & 82.92 & 87.56 & 133.56 & 132.94 & 133.25 & 213.90 & 215.04 & 214.47 \\
\hline Mean (A) & 85.38 & 77.18 & & 127.09 & 126.99 & & 212.51 & 213.32 & \\
\hline \multicolumn{10}{|l|}{$\mathbf{L S D}_{(0.05)}:$} \\
\hline (A) & & 4.20 & & & N.S. & & & N.S. & \\
\hline (B) & & 6.30 & & & 4.75 & & & 6.00 & \\
\hline$(\mathbf{A}) \times(B)$ & & 7.40 & & & 6.00 & & & 7.00 & \\
\hline \multicolumn{10}{|c|}{$2004 / 2005$} \\
\hline $\mathbf{Z n}_{0}+\mathbf{M n} \mathbf{n}_{0}$ & 84.98 & 79.00 & 81.99 & 118.35 & 119.00 & 118.68 & 206.17 & 203.31 & 204.74 \\
\hline $\mathbf{Z n}_{0}+\mathbf{M n}_{1}$ & 84.10 & 78.19 & 81.15 & 126.40 & 126.69 & 126.55 & 205.94 & 201.92 & 203.93 \\
\hline $\mathbf{Z n}_{0}+\mathbf{M n} \mathbf{n}_{2}$ & 83.72 & 77.80 & 80.76 & 135.63 & 135.18 & 135.41 & 202.68 & 200.14 & 201.41 \\
\hline $\mathbf{Z} \mathbf{n}_{1}+\mathbf{M n} \mathbf{n}_{0}$ & 92.18 & 86.40 & 89.29 & 117.49 & 117.81 & 117.65 & 210.15 & 206.68 & 208.42 \\
\hline $\mathbf{Z n}_{1}+\mathbf{M n} \mathbf{n}_{1}$ & 91.89 & 85.72 & 88.81 & 124.13 & 124.69 & 124.41 & 206.80 & 203.10 & 204.95 \\
\hline $\mathbf{Z n}_{1}+\mathbf{M n} \mathbf{n}_{2}$ & 91.28 & 85.24 & 88.26 & 130.88 & 131.28 & 131.08 & 203.98 & 201.69 & 202.84 \\
\hline $\mathbf{Z n}_{2}+\mathbf{M n}_{0}$ & 98.60 & 91.18 & 94.89 & 116.90 & 117.24 & 117.07 & 213.67 & 210.00 & 211.84 \\
\hline $\mathbf{Z n}_{2}+\mathbf{M n} \mathbf{n}_{1}$ & 97.89 & 90.69 & 94.29 & 125.00 & 125.43 & 125.22 & 211.94 & 207.94 & 209.94 \\
\hline $\mathbf{Z n}_{2}+\mathbf{M n}_{2}$ & 97.14 & 90.04 & 93.59 & 132.12 & 133.00 & 132.56 & 210.00 & 206.48 & 208.24 \\
\hline Mean (A) & 91.31 & 84.92 & & 125.21 & 125.59 & & 207.93 & 204.58 & \\
\hline
\end{tabular}

$\mathbf{L S D}_{(0.05)}$ :

\begin{tabular}{cccc}
$(\mathrm{A})$ & $\mathbf{4 . 5 0}$ & N.S. & N.S. \\
\hline$(\mathrm{B})$ & $\mathbf{6 . 7 0}$ & $\mathbf{6 . 1 5}$ & $\mathbf{5 . 7 0}$ \\
$(\mathrm{A}) \times(\mathrm{B})$ & $\mathbf{8 . 0 0}$ & $\mathbf{7 . 2 0}$ & $\mathbf{6 . 6 0}$
\end{tabular}

$\mathrm{Zn}_{0}=$ water (control), $\mathrm{Zn}_{1}=100 \mathrm{ppm}$, and $\mathrm{Zn}_{2}=150 \mathrm{ppm}$.

$\mathrm{Mn}_{0}=$ water (control), $\mathrm{Mn}_{1}=100 \mathrm{ppm}$, and $\mathrm{Mn}_{2}=150 \mathrm{ppm}$.

REFERENCES

Abed, T.A.; Zahi, M.E. and Abo-Sedera, F.A. (1987). Effect of some micronutrients foliar spraying and salinity stress on peas (Pisum sativum L.). I. Vegetative growth and chemical composition of plant foliage. Ann. Agric. Sci., Moshtohor, 25 (2): 1041-1056.

Amberger, A. (1978). Micronutrients, Dynamics in the soil and Function in plant metabolism. II. Manganese. Proc. Egypt. Bot. Soc. Workshop, Cairo,pp. 81-90.

Fayoum J. Agric. Res. \& Dev., Vol.19, No.2, July, 2005 
Mostafa M. Rady, et al.

Refaat, A.M. and Balbaa, L.K. (2001). Yield and quality of lemongrass plants (Cymbopogon flexuosus. Stapf) in relation to foliar application of some vitamins and microelements. Egypt. J. Hort., 28 (1): 41-57.

Bidwell, R.G.S. (1980). "Plant Physiology". $2^{\text {nd }}$ ed. Callier MacMillan Publisher London, New York.

Black, C.A. (1965). Methods of Soil analysis. Amer. Soc. Agron., Madison.

Broschat, T.K. and Moore, K.A. (2001). Influence of substrate and fertilizer analysis and rate of growth and quality of five species of bidding plants. Hort. Technology, 11 (3): 434-437.

Brown, J.D. and Lilliand, O. (1966). Rapid determination of potassium and sodium in plant material and soil extracts. Proc. Amer. Soc. Hort. Sci., 48: 341-346.

Chadha, A.P.S.; Rathore, S.V.S. and Ganeshe, R.K. (1999). Influence of N and $\mathrm{P}$ fertilization and ascorbic acid on growth and flowering of African marigold (Tagetes erecta L.). South Indian-Hort., 47 (1-6): 342-344.

Dahya, S.S.; Singh, N. and Singh, S. (1999). Effect of nitrogen and phosphorus on growth, flowering and yield of African marigold (Tagetes erecta L.). Environment and Ecology, 16 (4): 855-857.

Devlin, R.M. and Witham, F.H. (1985). Plant Physiology. CBS Publishers and Distributors, 485, Jain Bhawan, Bhola Nath Nagar, Shahdara, Delhi, 110032 (India).

El-Deeb, E.S.A. (1999). Effect of mineral nutrition, planting media and light on some foliage plants. M.Sc. Thesis, Fac. Agric., Cairo Univ., Egypt.

El-Gendy, S.A.; Hosni, A.M.; Ahmed, S.S. and Sabri, R.M. (2001). Sweet basil (Ocimum basilicum L.) productivity under different organic fertilization and inter plant spacing levels in a newly reclaimed land in Egypt. Sci., Cairo, 46 (1): 319-338.

Hafez, A. and Mikkelsen, D.S. (1981). Colorimetric determination of nitrogen for evaluating the nutritional status of rice. Comm. Soil Sci. and Plant Anal., 21 (1): 61-69.

Hameed, A.S. and Saker, K. (1999). Effect of graded levels of nitrogen and phosphorus on yield and quality of African marigold (Tagetes erecta L.). South Indian-Horticulture, 47 (1-6): 339-341.

Herbert, D.; Phipps, P.J. and Strange, R.F. (1971). Determination of total carbohydrates. Methods in Microbian, 5 (B): 209-344.

Kanwer, J.S. and Dhingra, F.R. (1962). Effect of micronutrients sprays on the chemical composition of citrates leaves and incidence of chlorosis. Ind. J. Agric. Sci., 32: 309-315.

King, E.J. (1951). Micro-analysis in medical biochemistry. $2^{\text {nd }}$ Ed., Churchil, London, UK.

Mengel, K. and Kirkby, E.A. (1982). Principles of Plant Nutrition. $3^{\text {rd }}$ Publisher International Potash Institute, Worblaven-Bern/Switzerland, pp. 1: 593-655.

Mohr, H. and Schopfer, P. (1995). "Plant Physiology". Translated Gudrum and Lawlor, D.V. Springer-Verlag, Berlin Heidelberg, New York.

Olsen, S.R. and Sommers, L.F. (1982). Phosphorus. In: Methods of Soil Analysis, Part 2, pp. 403-430. Am. Soc. Agron. Madison. W.I.

Fayoum J. Agric. Res. \& Dev., Vol.19, No.2, July, 2005 
EFFECT OF SOIL-N APPLICATION AND FOLIAR NUTRITION..... 95

Parkinson, J.A. and Allen, S.E. (1975). Awer oxidation procedure sutilable for the determination of nitrogen in mineral nutrients in biological material. Commun. Soil Sci. Plant anal., 17: 257-273.

Piper, C.S. (1947). "Soil and Plant Analysis'. The University of Adelaide, Adelaide, UK.

Price, C.A.; Clark, H.E. and Funkhauser, E.A. (1972). Function of micronutrients in plants. Soil Sci. of America, 231.

Ram, P.; Kumar, B.; Singh, A. and Yasseen, S. (1999). Response of African marigold (Tagetes erecta L.) to irrigation and nitrogen fertilization under Himalayan foot hills of Tarai region of Uttar Pradesh. Journal of Medicinal and Aromatic Plant Science, 21 (2): 361-366.

Selim, S.M. and El-Tantawy, A. (1993). Effect of some micronutrients on growth and flowering of gerbera plants. African J. Agric. Sd., Vol. 20 (2): 13-21.

Selim, S.M.; Hassanain, M.A. and EI-Sherif, M.M. (2001). Influence of zinc and manganese treatments on vegetative growth, flowering and chemical composition of Calendula officinalis plants. Annals Agric. Sci., Moshtohor, vol. 39 (3): 1713-1722.

Sillanpaa, M. (1982). Micronutrients and the nutrient status of soils: A global study, F.A.O. Soils Bull. 48, pp. 444.

Snedecor, G.W. and Cochran, W.G. (1980). "Statistical methods", $6^{\text {th }}$ ed., Iowa State Univ. Press. Amer., Iowa, U.S.A.

Vallee, B. and Walker, W.E.C. (1976). "CRC Handbook of Biochemistry and Molecular Biology" Proteins, vol.2.

Welburn, A.R. and Lichtenthaler, H. (1984). Formula and program to determine total carotenoids and chlorophyll $\mathrm{a}$ and $\mathrm{b}$ of leaf extracts in different solvents. In: "Advances in Photosynthesis Reseach" (Sybesma C.E.D.), Vol II, pp.9-12. Martinus Njihoff Dr.W. Junk Publ., The Hague.

Yadav, P.K.; Singh, S. and Dahiya, S.S. (1999). Effect of N and FYM on chlorophyll and nutrient in leaf of African marigold (Tagetes erecta L.) at flower bud initiation stage. Environment and Ecology, 17 (1): 188190.

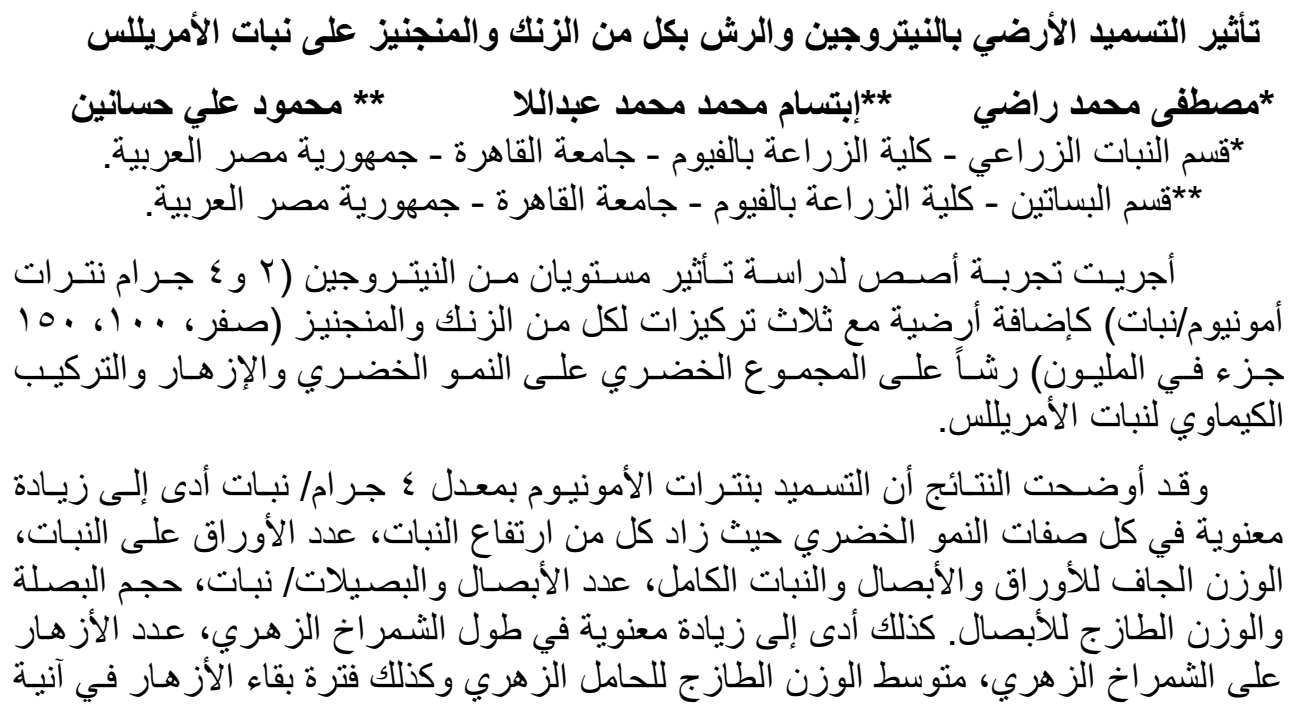

Fayoum J. Agric. Res. \& Dev., Vol.19, No.2, July, 2005 
Mostafa M. Rady, et al.

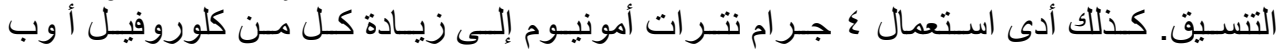

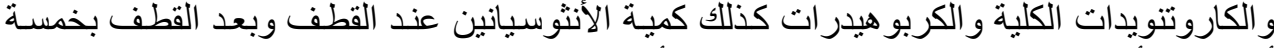

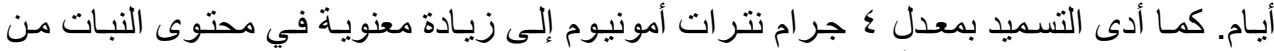

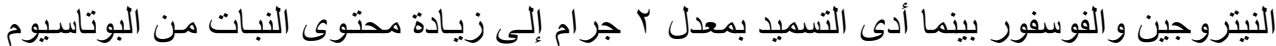

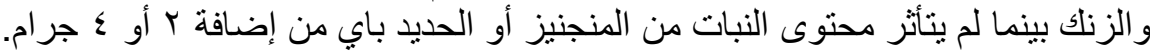

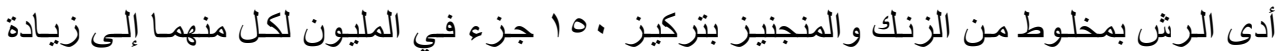

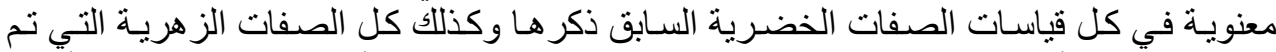

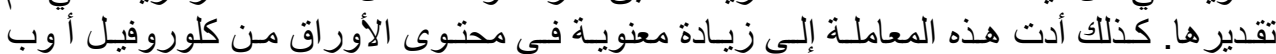

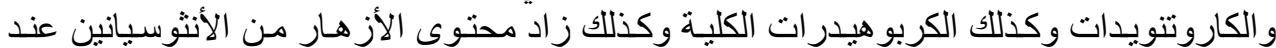

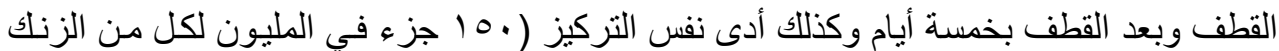

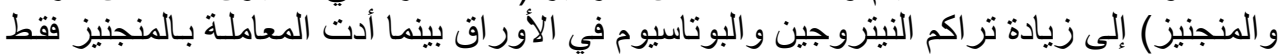

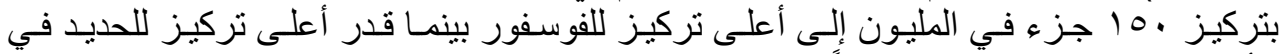

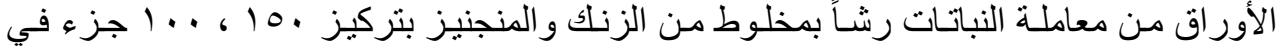

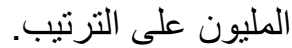

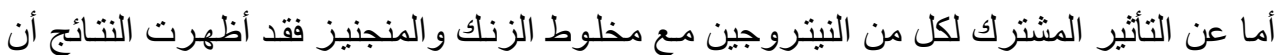

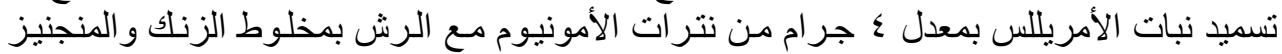

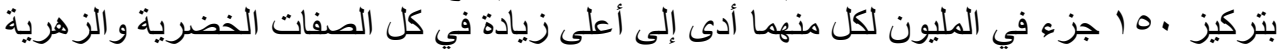

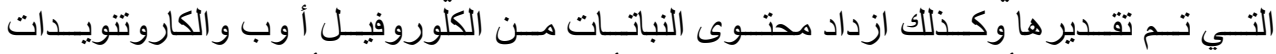

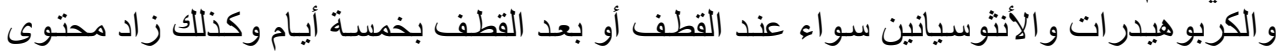

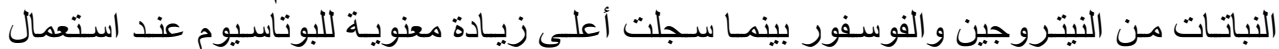

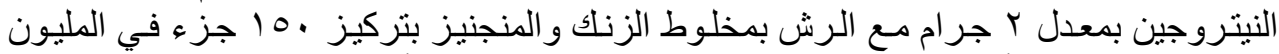

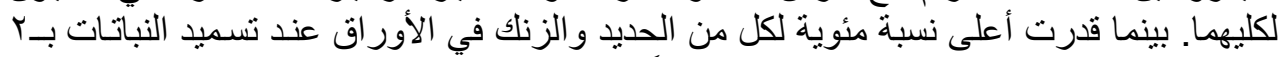

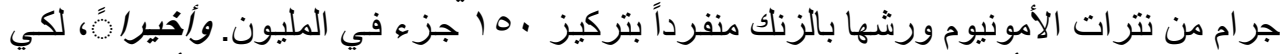

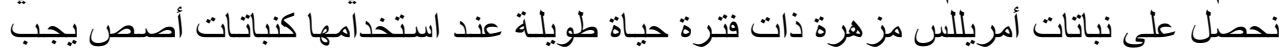

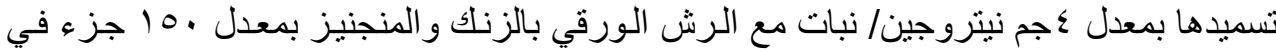

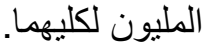

